\title{
Calculation of the Raman Line Broadening on Carbonation in Synthetic Hydroxyapatite
}

\author{
F. F. M. de Mul, ${ }^{*}$ C. Otto and J. Greve \\ University of Twente, Department of Applied Physics, P.O. Box 217, 7500 AE Enschede, The Netherlands
}

J. Arends and J. J. ten Bosch

State University of Groningen, Laboratory Materia Technica, A. Deusinglaan 1, 9713 AV Groningen, The Netherlands

\begin{abstract}
The position and broadening of the Raman band associated with the phosphate symmetric stretching vibration in hydroxyapatite are simulated using a simple inter- and intra-ionic potential. The results are compared with experimental values. This comparison was made as a function of the incorporation of carbonate ions in the lattice for a number of substitution models. The line width of the phosphate symmetric stretching vibration is shown both theoretically and experimentally to be dependent on the carbonate content. Good agreement between calculations and experiment is obtained. The results of the calculations offer some support to the suggestions in the literature that the dielectric constant has to be considered as a function of the distance in the lattice, increasing beyond the first environmental layer.
\end{abstract}

\section{INTRODUCTION}

Hydroxyapatite $\left[\mathrm{Ca}_{10}\left(\mathrm{PO}_{4}\right)_{6}(\mathrm{OH})_{2} ; \mathrm{HAP}\right]$ is a mineral closely related to the major mineral constituent of bone, dental enamel and other calcified tissues. The mineral may contain several kinds of impurities. A major impurity of inorganic origin is due to the incorporation of carbonate ions in the lattice. Typical values for the concentration of carbonate in the lattice are $0-20 \%$ and $2-5 \%$ in synthetic HAP and in sound dental enamel respectively. ${ }^{1,2}$ Hallsworth et al. ${ }^{3}$ suggested a relationship between the carbonate content of enamel and the susceptibility to dental caries.

In order to obtain some insight into the molecular composition of HAP on carbonation, we have performed micro-Raman spectroscopic measurements of HAP samples with varying carbonate concentration. Raman measurements were performed since the Raman spectrum is sensitive to the molecular rather than the atomic composition and samples with dimensions down to micrometre size may be studied. The spatial resolution is of the order of the wavelength of visible light. Measurements can be made in a non-destructive way, with the sample on a normal microscope slide. ${ }^{4}$

The results of Raman measurements on carbonated hydroxyapatite were published in a recent paper. ${ }^{5}$ The samples used were prepared at ambient temperature, using a method described by Arends et $a l^{6}{ }^{6}$ It was shown that the line width of the phosphate symmetric stretching vibration at $962 \mathrm{~cm}^{-1}$ is sensitive to carbonate contamination. From these measurements, it was concluded that the uptake of carbonate was accompanied by a line broadening of $0.90 \mathrm{~cm}^{-1}$ per $1 \%$ carbonate content, for carbonate contents smaller than $4 \%$. This was shown to be in accordance with measurements of line broadening by Nelson and Williamson, ${ }^{7}$ who obtained macroRaman spectra from carbonated samples prepared at high temperatures (about $1000^{\circ} \mathrm{C}$ ). However, at about $4 \%$ carbonate content a drastic change in the line

\footnotetext{
* Author to whom correspondence should be addressed.
}

broadening took place, from 0.90 to $0.10 \mathrm{~cm}^{-1}$ per $1 \%$ carbonate, indicating a deviation from the previously published results. ${ }^{7}$ The spectral position of the line $\left(962 \mathrm{~cm}^{-1}\right)$ did not change on carbonation. Also, the overall shape of the line remained unchanged as a nearly perfect Gaussian curve.

Some results of the measurements are displayed in Fig. 1, where the line width of the phosphate symmetric stretch vibration at $962 \mathrm{~cm}^{-1}$ is plotted as a function of the carbonate content. The drastic change in the increase of the line width with increasing carbonate content, at about $4 \%$ carbonate, can be seen. The measurements also revealed a similar change in the slope of the line broadening. with increasing carbonate content for the spectral line of the symmetric stretching vibration of the hydroxyl groups at $3575 \mathrm{~cm}^{-1}$.

A number of mechanisms may be responsible for these line broadening effects. The two most probable broadening mechanisms are the onset of re-orientational freedom of the phosphate groups in the lattice and a long-range disorder effect originally suggested by White. ${ }^{8}$ Both effects may be due to lattice disordering, caused by the creation of vacancies and other lattice disturbances accompanying the carbonate uptake.

In order to obtain some insight into the mechanisms responsible for the line broadening, we performed a model study of the phosphate vibration in the HAP lattice as a function of carbonate uptake. This was carried out for different carbonate substitution models. In addition, the influence of a possible contribution of re-orientational motions of the phosphate ions to the line broadening was also studied. The results are presented in this paper.

\section{THE HAP LATTICE AND CARBONATE SUBSTITUTION MODELS}

The lattice structure of HAP is hexagonal, with the lattice parameters $a$ and $b=0.9432 \mathrm{~nm}$ and $c=0.6881 \mathrm{~nm}$. At 


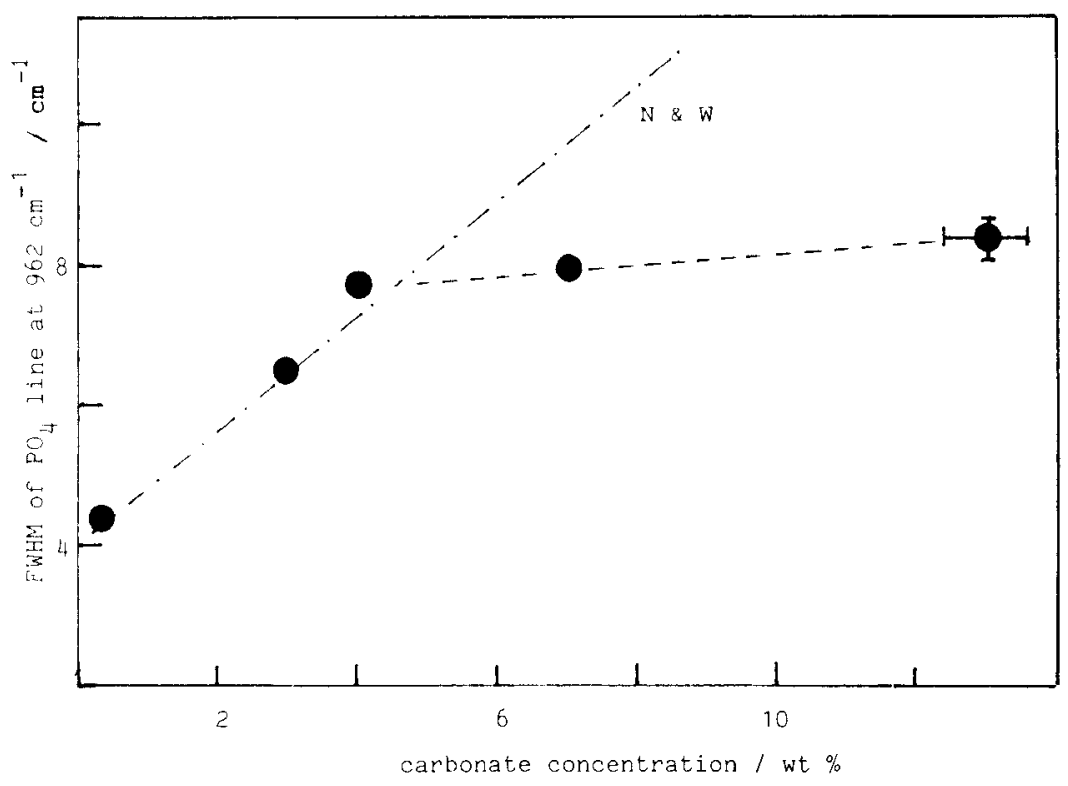

Figure 1. The full width at half-maximum intensity (FWHM) of the $\mathrm{PO}_{4}{ }^{3-} v$, line at $962 \mathrm{~cm}^{-1}$ as a function of the carbonate content (from Ref. 5). The error bars are typical for all points. For comparison, the calibration graph suggested by Nelson and Williamson $(N \& W)$ is included. ${ }^{7}$

$z=c / 4$ and $z=3 c / 4$ mirror symmetry planes are present. In Fig. 2 the HAP lattice is shown, where it can be seen that two different kinds of calcium ions are present: six ions constituting two equilateral triangles at $x=y=0$, and $z=c / 4$ and $3 c / 4$, respectively, and four ions in the channels formed by the phosphate ions, at $z=0$ and $c / 2$, respectively. The hydroxyl ions are positioned in the channels constituted by the $\mathrm{Ca}$ triangles. The $\mathrm{OH}$ axis is directed along the $c$-axis. For the orientation of the $\mathrm{OH}^{-}$ion two possibilities are suggested: ${ }^{9}$ the ordered column, in which all $\mathrm{OH}$-ions in a given column are oriented in the same way but with random choice of the direction of the orientation; and the disordered column, in which the direction of the $\mathrm{OH}^{-}$ions is reversed at various places within a particular column. A consequence of the latter is the creation of vacancies between $\mathrm{OH}$ and $\mathrm{HO}$, due to the actual position of those ions in the unit cell. In Table 1 the coordinates of the

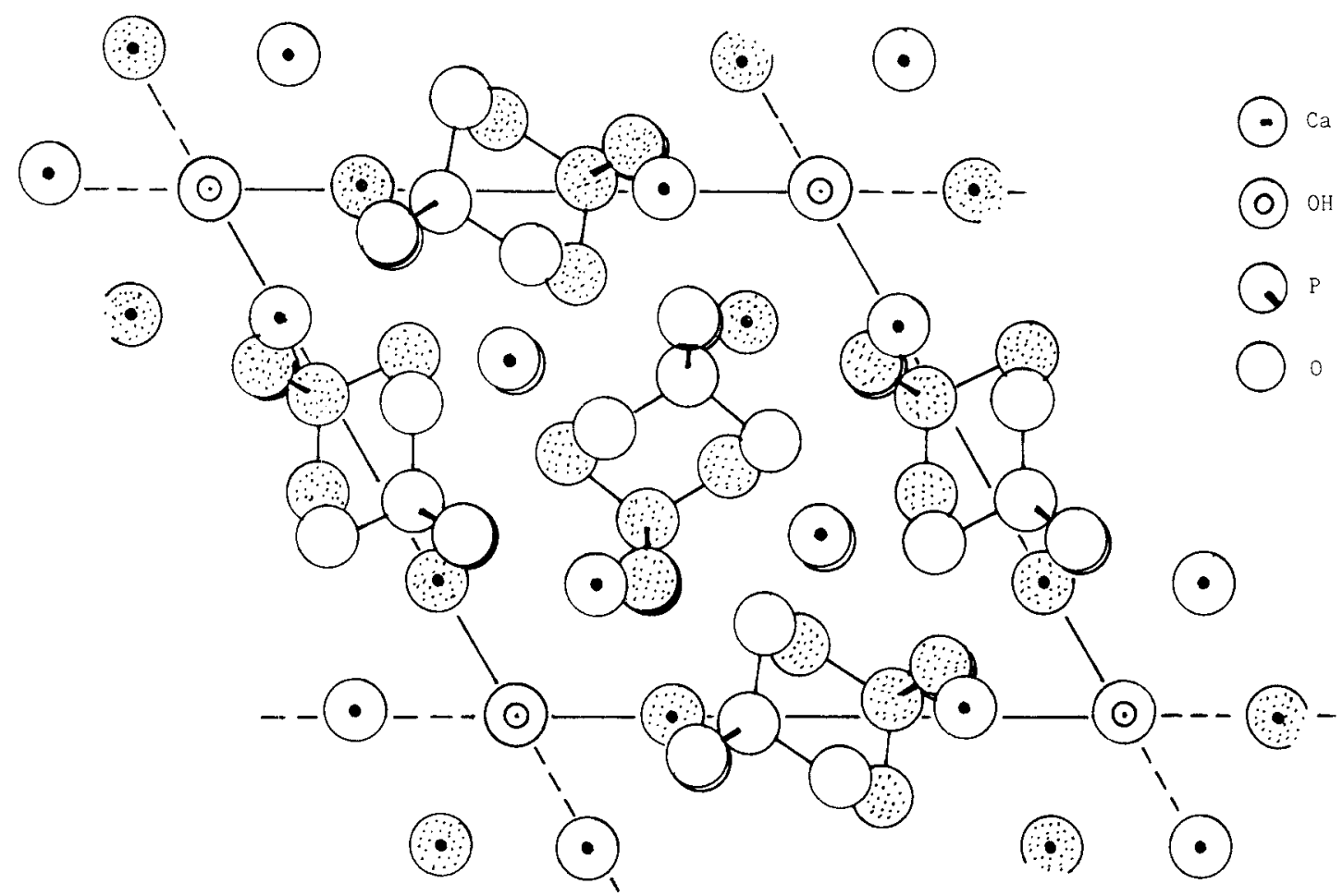

Figure 2. The HAP lattice: $x-y$ plane. The $c$-axis is perpendicular to this plane. The dotted atoms are positioned below $z / c=1 / 2$ 


\begin{tabular}{|c|c|c|c|c|}
\hline Ion & $x / a$ & $y / b$ & $2 / c$ & Remarks \\
\hline $\mathrm{Ca}$ & 0.3333 & 0.6667 & 0.0014 & Base for $4 \mathrm{Ca}$ atoms \\
\hline $\mathrm{Ca}$ & 0.2466 & -0.0069 & 0.2500 & Base for $6 \mathrm{Ca}$ atoms \\
\hline \multicolumn{5}{|l|}{$\mathrm{PO}_{4}:$} \\
\hline $\mathrm{P}$ & 0.3982 & 0.3682 & 0.2500 & \multirow{5}{*}{$\begin{array}{l}\text { base for } 6 \mathrm{PO}_{4} \\
\text { groups }\end{array}$} \\
\hline 0 & 0.3283 & 0.4846 & 0.2500 & \\
\hline 0 & 0.5876 & 0.4640 & 0.2500 & \\
\hline 0 & 0.3433 & 0.2579 & 0.0705 & \\
\hline 0 & 0.3433 & 0.2579 & 0.4295 & \\
\hline \multicolumn{5}{|l|}{$\mathrm{OH}$ : } \\
\hline (1) $\circ(a)$ & 0.0000 & 0.0000 & 0.2000 & \\
\hline (b) & - & - & 0.3000 & \\
\hline$H(a)$ & 0.0000 & 0.0000 & 0.0600 & \\
\hline (b) & - & - & 0.4400 & \\
\hline (2) $O(a)$ & 0.0000 & 0.0000 & 0.7000 & \\
\hline (b) & - & - & 0.8000 & \\
\hline$H(a)$ & 0.000 & 0.000 & 0.5600 & \\
\hline (b) & - & - & 0.9400 & \\
\hline
\end{tabular}

base atoms constituting the unit cell are given. From these the coordinates of the other atoms can be obtained by straightforward symmetry operations. In Table 1 the different possibilities for the coordinates and orientations of the $\mathrm{OH}^{-}$ions are also given. From x-ray work it was concluded that the root-mean-square deviation of the $\mathrm{OH}$ distance is $0.018 \mathrm{~nm}$, indicating a considerable freedom for translational motions of those ions along the $c$-axis of the lattice.

In HAP in crystalline form, the incorporation of carbonate ions has been suggested to take place at two different kinds of sites in the lattice (for a review, see Ref. 9), at the sites of $\mathrm{OH}^{-}$ions (A) and of $\mathrm{PO}_{4}{ }^{3-}$ ions (B). The A substitution can be performed at high temperatures (about $1000^{\circ} \mathrm{C}$ ), leading to A-type carbonate HAP ${ }^{10}$ while the B substitution takes place preferen- tially at ambient temperatures (B-type carbonate HAP). Both kinds of substitution are reversible. In dental enamel the incorporation of carbonate is assumed to occur primarily (about $95 \%$ ) at $\mathrm{PO}_{4}$ sites (B-type).

From $\mathrm{x}$-ray measurements it can be concluded ${ }^{9}$ that the $\mathrm{CO}_{3}$ substitutions are accompanied by changes in the $a$ parameter of the hexagonal HAP lattice. For each 1 wt $\%$ carbonate substituted, $\Delta a / a$ amounts to $25 \times$ $10^{-4}$ for the A-type and $-6 \times 10^{-4}$ for the B-type carbonate HAP. The $c$-axis of the lattice also shows a minor expansion. From ESR work it was concluded that 90 $95 \%$ of the carbonate ions in enamel turn up at $\mathrm{PO}_{4}$ positions, with the $\mathrm{C}_{3}$ axis of symmetry parallel to the lattice $c$-axis, and $5-10 \%$ at $\mathrm{OH}$ positions, with the $c_{3}$ axis perpendicular to the $c$-axis. ${ }^{11}$

For the incorporation of carbonate in HAP various models have been proposed:

I. First it has been assumed that on carbonate substitution three phosphate ions are replaced by four carbonate ions, leaving the oxygen content of the lattice unchanged, and introducing no vacancies. ${ }^{12-14}$ In order to maintain overall charge neutrality, it is necessary to introduce an $\mathrm{H}_{3} \mathrm{O}^{+}$ion at the position of the vanished $\mathrm{Ca}^{2+}$ ion (see Fig. 3):

$$
\mathrm{Ca}^{2+}+\mathrm{PO}_{4}{ }^{3-}+\mathrm{OH}^{-} \rightarrow \square_{\mathrm{Ca}}+\mathrm{CO}_{3}{ }^{2-}+\square_{\mathrm{OH}}
$$

Il. A second model has been proposed by several authors. ${ }^{15-17}$ In this model a $\mathrm{PO}_{4}$ group is replaced by a $\mathrm{CO}_{3}$ group, and vacancies at a neighbouring $\mathrm{Ca}$ ion and $\mathrm{OH}$ group are created:

$$
\mathrm{Ca}^{2+}+\mathrm{PO}_{4}{ }^{3-}+\mathrm{OH}^{-} \rightarrow \mathrm{Ca}_{\mathrm{Ca}}+\mathrm{CO}_{3}{ }^{2-}+\mathrm{O}_{\mathrm{OH}}
$$

It was assumed that this model implies the removal of the $\mathrm{OH}$ ion at $z / c=0.20-0.30$ and a neighbouring $\mathrm{Ca}$ ion at $z / c=1 / 4$, together with the substitution of the $\mathrm{PO}_{4}$ ion along the same direction by a $\mathrm{CO}_{3}$ ion, thus creating an oblong vacancy in the lattice.

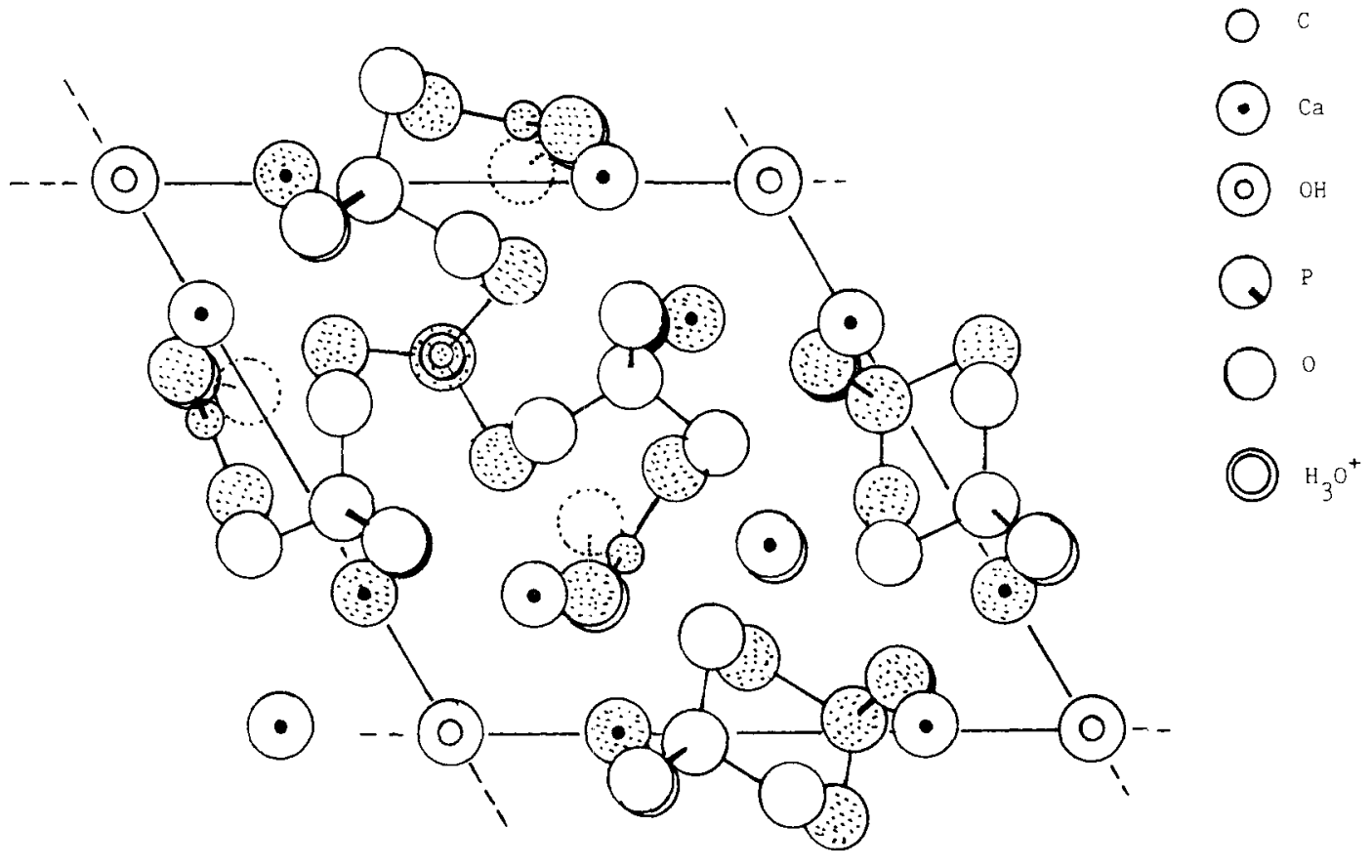

Figure 3. The HAP lattice with a substitution according to model I (see text). Three $\mathrm{PO}_{4}$ groups are transformed into $\mathrm{CO}_{3}$ groups. The remaining $\mathrm{O}$ atoms constitute the fourth $\mathrm{CO}_{3}$ group. A Ca ion has been replaced by an $\mathrm{H}_{3} \mathrm{O}^{+}$ ion. 
1II. A third model was proposed by Driessens et al.: ${ }^{18}$

$$
\mathrm{Ca}^{2+}+2 \mathrm{PO}_{4}{ }^{3-} \rightarrow \mathrm{Ca}_{\mathrm{C}}+2 \mathrm{CO}_{3}{ }^{2-}
$$

This can be achieved in different ways:

(a) $2 \mathrm{PO}_{4}$ ions at $z / c=1 / 4$ and $3 / 4$ in the same column, together with the $\mathrm{Ca}$ ion at $z / c=0$ or 0.5 ;

(b) $2 \mathrm{PO}_{4}$ ions at $z / c=1 / 4$ and $1 / 4$, or $3 / 4$ and $3 / 4$ in adjacent columns together with a $\mathrm{Ca}$ ion at $z / c=1 / 4$ or $3 / 4$.

IV. Another model was presented by Driessens et al.: ${ }^{18}$

$$
2 \mathrm{Ca}^{2+}+3 \mathrm{PO}_{4}{ }^{3-}+\mathrm{OH}^{--} \rightarrow 2-\mathrm{Ca}+3 \mathrm{CO}_{3}{ }^{2-}+\square_{\mathrm{OH}}
$$

Probably this model leads to a 'horizontal' layer of vacancies in a particular unit cell, consisting of $2 \mathrm{Ca}$ ions and $3 \mathrm{PO}_{4}$ ions originating from the layer at $z / c=$ $1 / 4$ or $3 / 4$.

From inspection of the consequences of the different models, it can be seen that model I will probably cause the least distortion in the lattice, in contrast to model IV.

The orientation of $\mathrm{CO}_{3}$ ions, when substituted for $\mathrm{PO}_{4}$ ions, is assumed to leave the position of the three $O$ atoms involved unchanged. The charge distribution may be taken as symmetrically distributed over the three $O$ atoms. However, when the carbonate ion is substituted for a hydroxyl group, which has its axis along the crystallographic $z$-axis, the symmetry axis of the $\mathrm{CO}_{3}$ ion may be assumed to be directed along the $z$-axis also. This is supported by the observation that the hydroxyl ion is positioned close to a mirror plane with a triangle of calcium ions. In this case the planar carbonate group still may have some translational freedom along the $z$-axis.

\section{THE INTERIONIC POTENTIAL}

For the potential function of the $\mathrm{P}-\mathrm{O}$ bond in the $\mathrm{PO}_{4}$ ions we have chosen a simple Lennard-Jones type of covalent potential, together with a Coulomb term in order to take the ionogenic interaction into account:

$$
V(r)=V_{0}\left[A\left(\frac{r_{0}}{r}\right)^{12}+B\left(\frac{r_{0}}{r}\right)^{6}\right]+\frac{z_{p} z_{0} e^{2}}{4 \pi \varepsilon r}
$$

In this expression $r$ measures the distance to the phosphorus atom of the relevant group; $V_{0}, A$ and $B$ are constants, to be specified later; $r_{0}$ is the equilibrium distance of minimum potential energy; and $z_{\mathrm{p}}$ and $z_{\mathrm{o}}$ represent the charge of the phosphorus and the oxygen atoms, respectively, corrected for the covalent binding. Instead of the Lennard-Jones potential other potential functions may be used. This will be considered later.

From this potential function the vibrational frequency follows in a straightforward manner:

$$
\omega_{0}^{2}=k / m ; \quad k=d^{2} V(r) / \mathrm{d} r^{2}
$$

According to the standard work of Pauling, ${ }^{19}$ for the case of a completely covalent $\mathrm{P}-\mathrm{O}$ bond, $z_{\mathrm{p}}$ and $z_{\mathrm{o}}$ should be +1 and -1 , respectively. However, the $\mathrm{P}-\mathrm{O}$ bond is assumed to have a bond number of 1.7 , indicating that a charge of $-0.7 e$ per $\mathrm{O}$ atom is transferred to the $\mathrm{P}$ atom. Taking into account a covalent fraction $c$ (or an ionogenic fraction $1-c)$, the transfer of charge from $\mathrm{O}$ to $\mathrm{P}$ will be $-1.7(1-c) e$. This leaves for the effective average charge of the phosphorus and the oxygen atoms

$$
z_{\mathrm{n}}=5.0-6.8 \mathrm{c} \text { and } z_{\mathrm{o}}=-2.0+1.7 \mathrm{c}
$$

From the electronegativity of the atoms it can be calculated that $c=0.61$, leading to $z_{\mathrm{p}}$ and $z_{o}=+0.85$ and -0.96 , respectively. However, we decided not to fix the value of $c$ and to fit it for the case of the undisturbed lattice, in order to have an intrinsic check on the validity of the model calculations. The value of $r_{0}$ was taken from crystallographic data: $r_{0}=0.154 \mathrm{~nm} .^{20}$

So far a free phosphate ion has been considered. Owing to the Coulomb interaction with ions surrounding the phosphate ion of interest, an interionic Coulomb term $V^{\prime}(r)$ should be added to the intra-ionic potential $V(r)$ (for notation see Fig. 4):

$$
V^{\prime}(r)=\sum_{v}\left(\frac{z_{\mathrm{o}} z_{v} e^{2}}{4 \pi \varepsilon r_{v o}}+\frac{z_{\mathrm{p}} z_{v} e^{2}}{4 \pi \varepsilon r_{v \mathrm{p}}}\right)
$$

In this expression the subscript $v$ indicates an environmental atom.

As a result of this interaction term, the minimum of the potential function will shift slightly from $r_{0}$ to $r_{01}$. This new equilibrium position will be given by

$$
\begin{array}{r}
V_{0}\left[-12 A\left(\frac{r_{0}}{r_{01}}\right)^{12}-6 B\left(\frac{r_{0}}{r_{01}}\right)^{6}\right] \\
-\frac{z_{\mathrm{p}} z_{\mathrm{o}} e^{2}}{4 \pi r_{01} \varepsilon}+\left.r_{01} \frac{\mathrm{d} V^{\prime}(r)}{\mathrm{d} r}\right|_{r_{01}}=0
\end{array}
$$

Since $r_{01}$ will be close to $r_{0}$, we assume that it is allowed to use a Taylor expansion to a first approximation:

$$
\begin{aligned}
& \left.\frac{\mathrm{d} V^{\prime}(r)}{\mathrm{d} r}\right|_{r_{01}}=\left.\frac{\mathrm{d} V^{\prime}(r)}{\mathrm{d} r^{2}}\right|_{r_{0}}+\left.\frac{\mathrm{d}^{2} V^{\prime}(\Gamma)}{\mathrm{d} r^{2}}\right|_{r_{0}}\left(r_{01}-r_{0}\right) \\
& V_{0}\left[12 A\left(\frac{r_{0}}{r_{01}}\right)^{12}+6 B\left(\frac{r_{0}}{r_{01}}\right)^{6} \frac{1}{r_{01}}\right. \\
& +\frac{z_{\mathrm{p}} z_{0} e^{2}}{4 \pi \varepsilon r_{01}^{2}}-\left.\frac{\mathrm{d} V^{\prime}(r)}{\mathrm{d} r}\right|_{r_{0}}-\left.\frac{\mathrm{d}^{2} V^{\prime}(r)}{\mathrm{d} r^{2}}\right|_{r_{0}}\left(r_{01}-r_{0}\right)=0
\end{aligned}
$$

This equation has to be solved numerically. With the new equilibrium position $r_{01}$ the new value for the force constant $k^{\prime}$ and the new value of the frequency $\omega^{\prime}$ of the

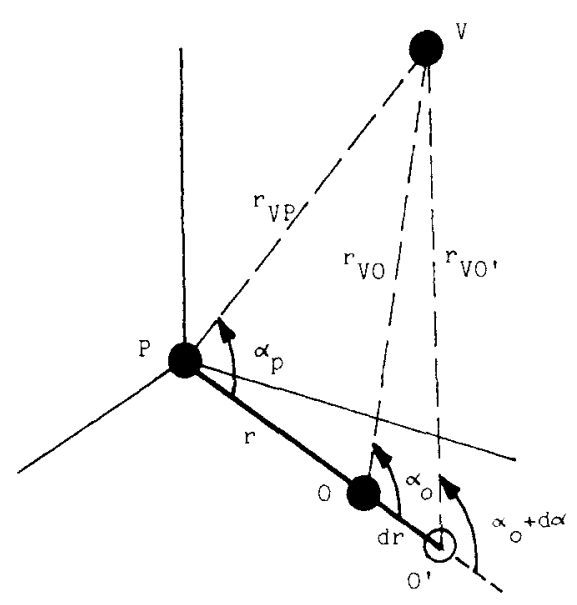

Figure 4. Calculation of the potential and its derivatives, as a function of the P-O distance $r$. The subscript $v$ denotes an environmental atom. 
vibration can be calculated using

$$
\left(\omega^{\prime}\right)^{2}=k^{\prime} / m ; \quad k^{\prime}=d^{2}\left(V+V^{\prime}\right) / \mathrm{d} r^{2}
$$

which leads to

$$
\begin{aligned}
k^{\prime} & =V_{0}\left[156 A\left(\frac{r}{r_{01}}\right)^{12}+42 B\left(\frac{r_{0}}{r_{01}}\right)^{6}\right] \\
& +\frac{2 z_{\mathrm{p}} z_{\mathrm{o}} e^{2}}{4 \pi \varepsilon r_{01}{ }^{3}}+\left.\frac{\mathrm{d}^{2} V^{\prime}}{\mathrm{d} r^{2}}\right|_{r_{01}}
\end{aligned}
$$

where

$$
\left.\frac{\mathrm{d}^{2} V^{\prime}(r)}{\mathrm{d} r^{2}}\right|_{r_{01}}=\left.\frac{\mathrm{d}^{2} V^{\prime}(r)}{\mathrm{d} r^{2}}\right|_{r_{0}}+\left.\frac{\mathrm{d}^{3} V^{\prime}(r)}{\mathrm{d} r^{3}}\right|_{r_{0}}\left(r_{01}-r_{0}\right)
$$

and (see Fig. 4)

$$
\begin{gathered}
\mathrm{d} V^{\prime} / \mathrm{d} r=C_{0} \cos \alpha_{0} / r_{v o}^{2} \\
\mathrm{~d}^{2} V^{\prime} / \mathrm{d} r^{2}=C_{0}\left(3 \cos \alpha_{0}-1\right) / r_{v o}^{3} \\
\mathrm{~d}^{3} V^{\prime} / \mathrm{d} r^{3}=C_{0}\left(15 \cos ^{3} \alpha_{0}-9 \cos \alpha_{0}\right) / r_{v o}^{4} ; \\
C_{0}=\frac{z_{\mathrm{p}} z_{\mathrm{o}} e^{2}}{4 \pi \varepsilon}
\end{gathered}
$$

This calculation has to be carried out for the P-O bonds in all phosphate ions in a representative region of the lattice (with periodic boundary conditions), each having an environment that has to be chosen to be sufficiently large. The resulting set of corrected frequencies will build up the spectral line, which will be broadened, especially in the case of local differences due to lattice distortions.

\section{CALCULATIONS}

\section{Phosphate ion reorientations}

From basic Raman theory (for a review see, e.g., Ref. 21 ), it follows that the contribution to the line width from reorientational motions can be obtained by comparison of the Fourier transforms of the spectra of the polarized and the depolarized Raman scattering, corrected for a possible contribution to the broadening by vibrations and for the instrumental spectral resolution. The polarized and depolarized scattering functions $I_{\text {pol }}$ and $I_{\mathrm{dep}}$ are given by

$$
\begin{aligned}
I_{\text {pol }}(\omega) & =I_{\text {iso }}(\omega)+(4 / 3) I_{\text {depol }}(\omega) \\
I_{\text {iso }}(\omega) & =I_{\text {vib }}(\omega) * I_{\text {trans }}(\omega) * R(\omega) \\
I_{\text {depol }}(\omega) & =I_{\text {vib }}(\omega) * I_{\text {trans }}(\omega) * I_{\text {rot }}(\omega) *(\omega)
\end{aligned}
$$

where $I_{\text {trans }}, I_{\mathrm{vib}}$ and $I_{\text {rot }}$ are the scattering functions due to translational, vibrational and rotational motions, respectively, $R(\omega)$ is the experimental resolution function and * represents a convolution. By Fourier transformation of eqns (13) and (14), followed by a division, the rotational function can be obtained. Subsequently, from $I_{\text {rot }}(t)$ a rotational correlation function can be derived.

From the measurements, ${ }^{5}$ it was concluded that the depolarization ratio $\left[I_{\mathrm{dep}}(\omega) / I_{\text {pol }}(\omega)\right]$ equals $0.09 \pm 0.01$. Further, the widths of $I_{\text {pol }}(\omega)$ and $I_{\text {dep }}(\omega)$ turned out to be equal, within the limits of error. This means that there is only a small contribution from rotations, with a slow time scale, larger than 100 ps. For comparison, the time scale for free rotations of a phosphate-like molecule is about 2 ps. The non-zero result for the depolarization ratio also indicates a slight distorsion of the $\mathrm{PO}_{4}$ tetraeder, leading to a lower symmetry than $T_{d}$ (probably $C_{3 v}$ ), since a completely symmetric vibration like the ideal $\mathrm{PO}_{4}$ ion is not sensitive to rotational motions. Therefore, in eqns (13) and (14) $I_{\text {rot }}(\omega)$ may be replaced by a delta-function in $\omega$.

The translational contribution can also be approximated with $\delta(\omega)$, since in the expression for $I_{\text {trans }}$ on time scale:

$$
I_{\text {trans }}(t)=\langle\exp \{-\mathbf{q} \cdot[\mathbf{r}(t)-\mathbf{r}(0)]\}\rangle
$$

the inverse of the wavenumber $q$ is much larger than the deviation of the centre of mass of the phosphate ion, given by $r(t)-r(0)$, leading to $I_{\text {trans }}(t)=1$.

Thus we may write for Eqns (12) and (13):

$$
I_{\text {pol }}(\omega)=I_{\text {iso }}(\omega)=I_{\text {vib }}(\omega) * R(\omega)
$$

Both the measured lines and the instrumental resolution can be represented by Gaussian curves to a good approximation, so facilitating the correction of $I_{\mathrm{vib}}(\omega)$ for $R(\omega)$. The resolution function had a full width at half maximum intensity (FWHM) of $3.2 \mathrm{~cm}^{-1}$ during all measurements.

From the considerations given above we may conclude that the most significant contribution to the line broadening is due to vibrational effects.

\section{Potential distortions due to carbonate substitution}

To calculate the influence of the substitution of carbonate ions in the lattice, a computer program which could deal with different kinds of substitution was developed. In the calculations use was made of a base set of $2 \times 2 \times 2$ unit cells of the HAP lattice, together with an environment defined using periodic boundary conditions. This set of 8 cells contains 48 phosphate ions or $192 \mathrm{P}-\mathrm{O}$ bonds. In the calculations each cell is embedded in the centre of a cube of $n \times n \times n$ unit cells. This environment is used to calculate the correction $V^{\prime}(r)$ on the potential function, as described under The Interionic Potential, by summation over the contribution of all individual atoms to the potential energy of all 192 individual $\mathrm{P}-\mathrm{O}$ bonds in the base set. Changes in the content of each cell of the base set, and in the positions of the atoms contained in the cell, corresponding to an individual substitution or to a set of substitutions, as with the models described, can be performed easily. Using periodic boundary conditions those changes are multiplied throughout the lattice. In this way, for instance, an overall carbonate substitution can be simulated. Then, for each $\mathrm{P}-\mathrm{O}$ bond of each phosphate ion in the base set the corrected $\mathrm{P}-\mathrm{O}$ distance is calculated. This is done by solving Eqn (7) using a numerical method of zero-order approximation. Using these corrected distances for each phosphate group, a corrected value for the phosphate line frequency is calculated. The spectral line width is obtained from the standard deviation of the distribution of all corrected frequencies.

The program also offers the possibility of a normalization of all $\mathrm{P}-\mathrm{O}$ distances to a value to be chosen freely, 
or the incorporation of a parallel or an anti-parallel ordering of the hydroxyl ions (as described under The HAP Lattice and Carbonate Substitution Models). To study the translational freedom of the hydroxyl ions, a shift in the positions of those ions can be introduced.

To start the calculations some constants in the equations still have to be defined. For the dielectric constant the vacuum value may be taken. This is probably correct for the nearest neighbours, but becomes dubious for the outer shells. This matter will be discussed later. The best fit to the experimental results was obtained with the constants $A$ and $B$ taken as equal to $+c$ and $-c$, respectively, especially for the values of the spectral line position and the line width at zero carbonate content (the undisturbed lattice). The value of $c$ was not taken from the value calculated by Pauling, but obtained by fitting the spectral line position of the undisturbed lattice, as obtained from the calculations, to the experimental value, using the value of $r_{0}$ as the only input constant.

In principle, the values of $V_{0}, A, B$ and $c$ should also follow from experimental data with free phosphate ions. In the literature some divergent values for the freephosphate frequency have been published: $938,{ }^{22}, 980^{23}$ and $1003-1006 \mathrm{~cm}^{-1} .24$ Moreover, it turns out that most of these values have been obtained from measurements on phosphate ions in a solution, especially aqueous. Therefore, we decided not to take those values, but to calculate the potential constant $V_{0}$ from the experimental value obtained for the frequency of the undisturbed lattice in a direct way, together with the value of $c$ and the frequency for really free phosphate using the potential function given in Eqn (1). Putting the first and second derivative of $V(r)$ equal to 0 and $k$, respectively, results in

$$
\begin{gathered}
V_{0}=-C_{0} /\left(12 A r_{0}+6 B r_{0}\right) ; \\
k=\left[V_{0}(156 A+42 B)+2 C_{0} / r_{0}\right] / r^{2}
\end{gathered}
$$

With the value of $r_{0}$ as mentioned before and with $c$ as a parameter, the line positions shown in Fig. 5 were obtained. Then, using the experimental value of $962 \mathrm{~cm}^{-1}$ for the phosphate line, for $c$ a value of 0.602 is obtained and for $V_{0}$ a value of $2.29 \mathrm{eV}$. The freephosphate frequency turns out to be $1004 \mathrm{~cm}^{-1}$. A change in $r_{0}$ of $0.0015 \mathrm{~nm}(1 \%)$ was calculated to result in a change in frequency of about $15 \mathrm{~cm}^{-1}(1.5 \%)$. Typical experimental values for the frequency shifts are

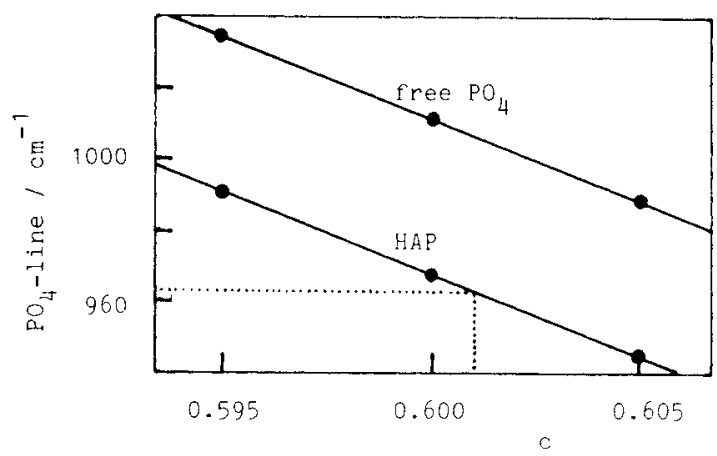

Figure 5. The $\mathrm{PO}_{4}$ line frequency, calculated as a function of the covalent fraction $c_{\text {, }}$ for HAP and for free $\mathrm{PO}_{4}$ ions, with $r_{0}=0.154 \mathrm{~nm}$. The experimental value $\left(962 \mathrm{~cm}^{-1}\right)$ is indicated also, together with the resulting $c$ value

$4-10 \mathrm{~cm}^{-1}$. The values of $c$ and $\omega$ obtained in this way are in good agreement with the values given by Pauling ${ }^{19}$ and Nishino et al., ${ }^{24}$ respectively.

In these calculations, the effect of the dimensions of the environmental cube of $n \times n \times n$ unit cells surrounding each base set cell was investigated and $n=5$ was found to be a good compromise between accuracy and a reasonable computing time. For $n=6$ no significant improvement in accuracy was found. Therefore, in all subsequent calculations a value of $n=5$ was taken, and no ions at a distance from the particular phosphate ion of relevance exceeding $2 \mathrm{~nm}$ were taken into account.

The calculations were carried out on a PDP-LSI 11/73 computer. Each cycle of frequency calculations following a particular substitution, as mentioned under The HAP Lattice and Carbonate Substitution Models, took about $30 \mathrm{~min}$. In order to check the validity of the method we calculated for the undisturbed lattice the spread in frequency of the phosphate line on a slight shift of the hydroxyl ions from their initial positions as derived from the crystallographic data, both for parallel and anti-parallel orientations of the hydroxyl ions in adjacent columns in the lattice. The results are displayed in Fig. 6. In these calculations a slightly different value for $c, 0.600$, causing shifted values for the line frequency also, was used. From Fig. 6 it can be seen that the experimental value for the line broadening for the undisturbed lattice, $4.1 \pm 0.1 \mathrm{~cm}^{-1}$ (see Fig. 1), was reproduced fairly well by the calculated values.

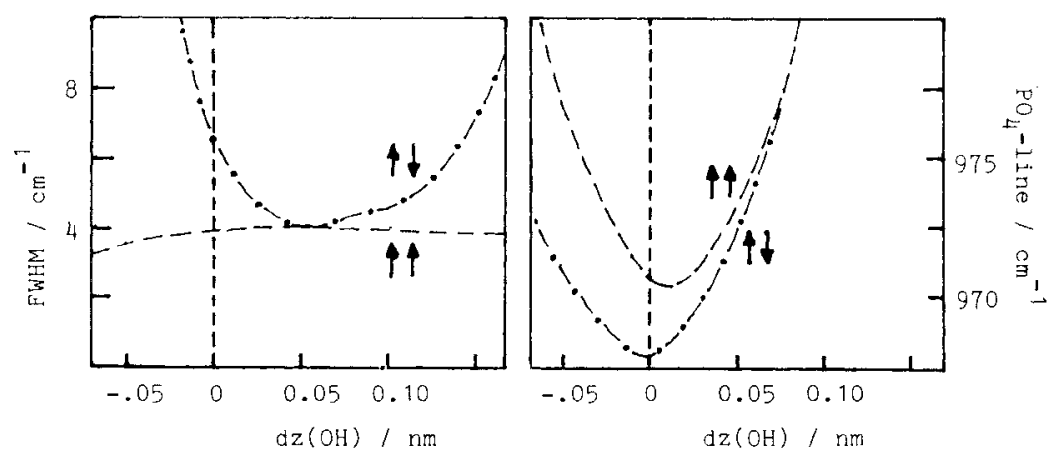

Figure 6. (a) The $\mathrm{PO}_{4}$ line broadening and (b) the line frequency, calculated as a function of the deviation $d z$ of the hydroxyl positions from the $x$-ray values. These values are calculated for parallel and anti-parallel orientation of the hydroxyl ions in adjacent columns in the lattice. $r_{0}=0.154 \mathrm{~nm}$ and $c=0.600$. 
Table 2. Carbonate substitutions

\begin{tabular}{|c|c|c|c|}
\hline Series & Model & Cell No. & Substitution \\
\hline \multirow[t]{5}{*}{1} & 1 & 1 & $\mathrm{PO}_{4}$ at pos. $36 \rightarrow \mathrm{CO}_{3} ; \mathrm{O}$ at $37 \rightarrow$ vac. \\
\hline & & 3 & $\mathrm{PO}_{4}$ at pos. $26 \rightarrow \mathrm{CO}_{3} ; \mathrm{O}$ at $27 \rightarrow$ vac. \\
\hline & & 1 & $\mathrm{PO}_{4}$ at pos. $11 \rightarrow \mathrm{CO}_{3} ; 0$ at $12 \rightarrow$ vac. \\
\hline & & 1 & $\mathrm{Ca}$ at pos. $1 \rightarrow \mathrm{CO}_{3}(z=c / 4)$ \\
\hline & & 1 & $\mathrm{Ca}$ at pos. $1 \rightarrow \mathrm{H}_{3} \mathrm{O}^{+}$ \\
\hline \multirow[t]{2}{*}{$2-5$} & II & 1 & $\mathrm{OH}$ at pos. $41 \rightarrow$ vac.; $\mathrm{Ca}$ at $5 \rightarrow$ vac. \\
\hline & & 1 & $\begin{array}{l}\mathrm{PO}_{4} \text { at pos. } 26 \rightarrow \mathrm{CO}_{3} ; \mathrm{O} \text { at } 27-30 \rightarrow \\
\text { vac. }\end{array}$ \\
\hline \multirow[t]{3}{*}{$6-9$} & Illa & 1 & $\mathrm{Ca}$ at pos. $4 \rightarrow$ vac. \\
\hline & & 1 & $\begin{array}{l}\mathrm{PO}_{4} \text { at pos. } 21 \rightarrow \mathrm{CO}_{3} ; \mathrm{O} \text { at } 22-25 \rightarrow \\
\text { vac. }\end{array}$ \\
\hline & & 1 & $\begin{array}{l}\mathrm{PO}_{4} \text { at pos. } 26 \rightarrow \mathrm{CO}_{3}: \mathrm{O} \text { at } 27-30 \rightarrow \\
\text { vac. }\end{array}$ \\
\hline \multirow[t]{3}{*}{10} & IIIb & 1 & $\mathrm{Ca}$ at pos. $5 \rightarrow$ pos. $5 \rightarrow$ vac. \\
\hline & & 1 & $\mathrm{PO}_{4}$ at pos. $11 \rightarrow \mathrm{CO}_{3} ; \mathrm{O}$ at $12 \rightarrow$ vac. \\
\hline & & 1 & $\mathrm{PO}_{4}$ at pos. $26 \rightarrow \mathrm{CO}_{3} ; \mathrm{O}$ at $28 \rightarrow$ vac. \\
\hline \multirow[t]{2}{*}{$11-14$} & IIIC & 1 & As in 10 \\
\hline & & 1 & $\begin{array}{l}O \text { at pos. } 13+27,14+29,15+30,15+ \\
29 \rightarrow \text { vac. }\end{array}$ \\
\hline \multirow[t]{4}{*}{15} & & 1 & $\mathrm{Ca}$ at pos. $5,6 \rightarrow$ vac.; $\mathrm{OH}$ at $41 \rightarrow$ vac. \\
\hline & & 1 & $\mathrm{PO}_{4}$ at pos. $26 \rightarrow \mathrm{CO}_{3} ; \mathrm{O}$ at $29 \rightarrow$ vac. \\
\hline & & 1 & $\mathrm{PO}_{4}$ at pos. $11 \rightarrow \mathrm{CO}_{3} ; \mathrm{O}$ at $14 \rightarrow$ vac. \\
\hline & & 1 & $\mathrm{PO}_{4}$ at pos. $31 \rightarrow \mathrm{CO}_{3} ; \mathrm{O}$ at $39 \rightarrow$ vac. \\
\hline
\end{tabular}

In Table 2 a survey is given of the substitutions for which frequency spreads were calculated. Figure 7 shows the notation to be used with Table 2 . The substitutions were performed for cell No. 1 of the base set. As a result of the periodicity in the cells the same substitutions were performed in equivalent cells in adjacent base sets of cells. A similar set of calculations were performed with substitutions, not only in cell No. 1, but also in cell No. 5 of the base set of cells. Also, the relative orientation of the OH columns (parallel/anti-parallel) was varied, together with the deviation of the $\mathrm{OH}$ ions from their $\mathrm{x}$-ray position (as described before). In the substitutions the $\mathrm{C}-\mathrm{O}$ distances were renormalized to $0.130 \mathrm{~nm}$, as given by several authors (e.g. Pauling. ${ }^{19}$ ).

Instead of the Lennard-Jones type of potential, other potential functions can also be used. However, applying the frequently used Born-Mayer potential we could not obtain a significantly better fit, at least for the undisturbed lattice with no carbonate. We did not apply this type of potential to the substituted lattices. Nevertheless, since the effect of the substitutions turns out to be only a slight perturbation of the undisturbed lattice, to a first approximation the results for the extra line broadening on carbonation should be comparable.

\section{RESULTS OF THE COMPUTATIONS}

With all models described in the previous section the position of the phosphate line remains the same, independent of the carbonate content, within the limits of error (about $\pm 1 \mathrm{~cm}^{-1}$ ). The line width, however, was sensitive to changes in the carbonate concentration, i.e. for the various models in a different way. Also, the line broadening was sensitive for changes in the dielectric constant.

Table 3 shows some results schematically. The limits of error were calculated from the spread of the results obtained for the various submodels of the substitutions given in Table 2 . The results for the various submodels are comparable. This, however, is not always the case for the actual line shape. In Fig. 8 a survey of different line shapes is given for one of the models.

The results in Table 3 were obtained with the dielectric constant equal to the vacuum value. Although the calculated results are of the same order as the experimental values, the agreement is not perfect. A possible explanation for the calculated values being too large may be the assumption of the vacuum dielectric constant throughout the lattice, independent of distance.

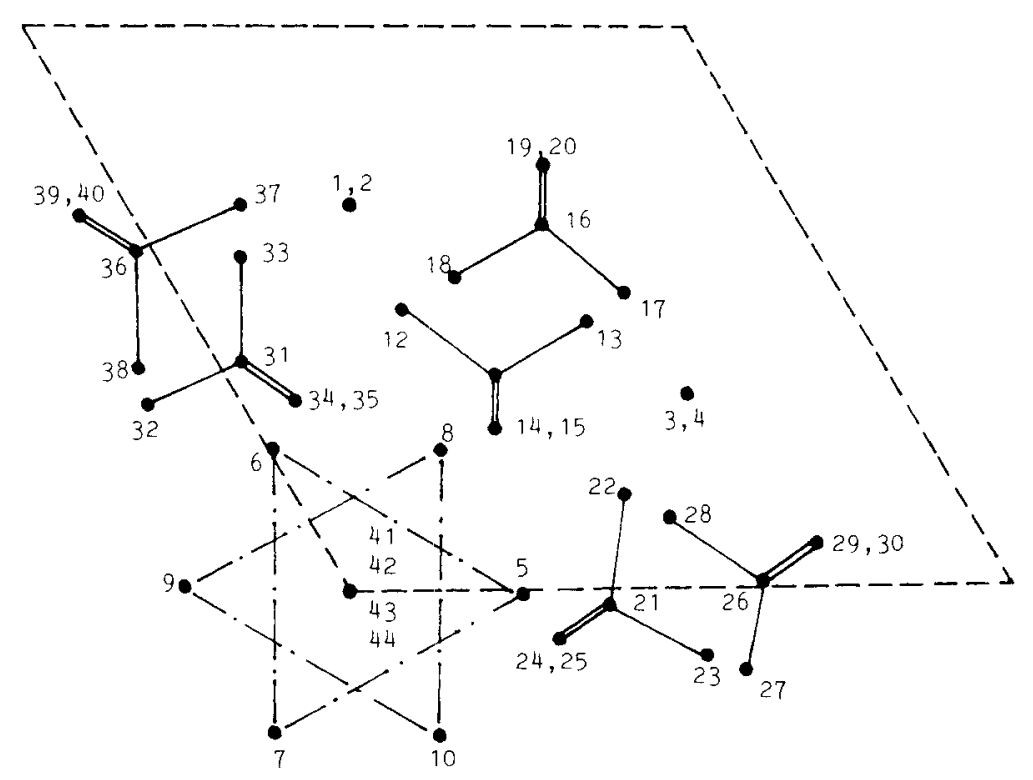

Figure 7. Numbering of atoms in the unit cell of the HAP lattice. These numbers are to be used with Table 2, together with Fig. 1 and Table 1 . The Ca ions 1, 2 3 and 4 are at $z / c=0,1 / 2,0$ and $1 / 2$, respectively. The $O H$ ions with Nos $41-42$ and $43-44$ are below and above $z / c=1 / 2$, respectively. 
Table 3. Calculated phosphate line broadening $\left(\varepsilon_{r}=1\right)$

\begin{tabular}{|c|c|c|c|c|c|c|}
\hline \multirow{2}{*}{$\begin{array}{l}\text { Substitution } \\
\text { model }\end{array}$} & \multicolumn{2}{|c|}{$\mathrm{CO}_{3}(\%)$} & \multicolumn{2}{|c|}{$\Delta(F W H M)\left(\mathrm{cm}^{-1}\right\}$} & \multicolumn{2}{|c|}{$\begin{array}{c}\Delta(\mathrm{FWHM}) / \% \mathrm{CO}_{3} \\
\left(\mathrm{~cm}^{-1 / \%} / \%\right)\end{array}$} \\
\hline & a & b & a & b & a & b \\
\hline 1 & 3.0 & 6.1 & 0.9 & 0.7 & 0.3 & $0.1^{\mathrm{a}}$ \\
\hline II & 0.8 & 1.5 & $3.6 \pm 0.5$ & $5.4 \pm 0.8$ & $4.5 \pm 0.6$ & $3.6 \pm 0.6$ \\
\hline IIla & 1.5 & 3.1 & $2.6 \pm 0.5$ & $5.7 \pm 0.8$ & $1.7 \pm 0.4$ & $1.8 \pm 0.3$ \\
\hline $111 \mathrm{~b}$ & 1.5 & 3.1 & $4.1 \pm 0.7$ & $5.8 \pm 0.8$ & $2.7 \pm 0.5$ & $1.9 \pm 0.3$ \\
\hline IV & 2.3 & 4.6 & 4.5 & 7.0 & 2.0 & $1.5^{\mathrm{a}}$ \\
\hline Exp. & \multicolumn{2}{|c|}{$>4.0$} & & & \multicolumn{2}{|c|}{$0.10 \pm 0.10$} \\
\hline
\end{tabular}

${ }^{a}$ Line shapes consisting not of a single line but of two or more adjacent and fairly separated lines.

However, it has been suggested ${ }^{25-27}$ that for distances larger than that corresponding to the first environmental layer a relative dielectric constant larger than 1 should be used. The dielectric constant should approach the macroscopic bulk value for large distances, but this bulk dielectric constant is not known for HAP. This suggestion was tested in molecular dynamics calculations on some hydrocarbon lattices. ${ }^{28}$ We repeated the calculations for various regimes for the dielectric constant as a function of the distance. The calculations were carried out with the dielectric constant increasing slowly with distance, with various slopes, and also for a sudden change in the dielectric constant at a distance just outside the first environmental layer as seen from a phosphate ion.

From these calculations it followed that not only does the extra line broadening on carbonation change with increasing dielectric constant, but also the line width

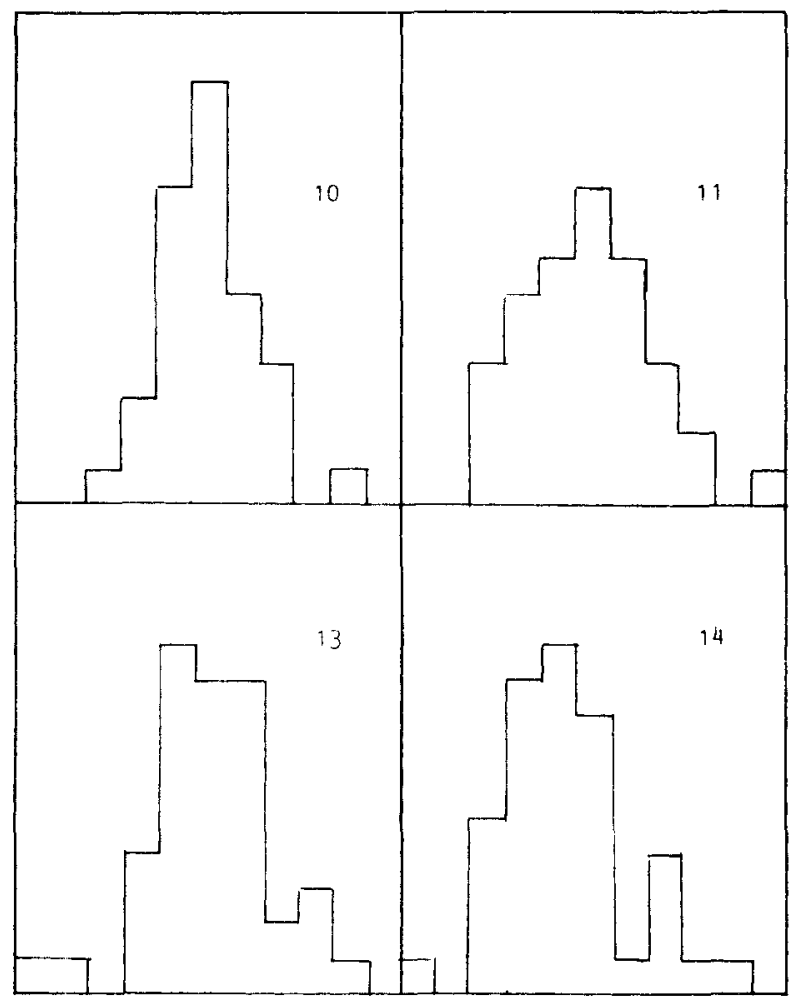

Figure 8. Typical line shapes, calculated for model IIlb (submodels $10,11,13$ and 14), corresponding to Table 3 . Horizontal scale: $2 \mathrm{~cm}^{-1}$ per division. value for the undisturbed lattice. Again, the line position remained remarkably constant. In Table 4 some results of these calculations are summarized.

A comparison of the results in Table 4 with those in Table 3 shows that the best agreement with the experimental data is obtained for the two regimes indicated in Table 4 under $\alpha$. The results of models I and II are insensitive to changes in the dielectric constant, whereas models IIIa and IIIb show an increasing and a decreasing behaviour, respectively, with increasing dielectric constant. Model IV is uncertain.

\section{DISCUSSION}

The results are in agreement with what might be expected for the various models on the basis of the relative impact that these models have on the structure of the lattice. With model I the lattice remains the most undisturbed on replacement of phosphate ions by carbonate ions. This is reflected in a small value for the slope of the extra line broadening per $1 \%$ carbonate, while the other models, in which vacancies are created, show a much larger slope. Probably model I accounts for the mechanism of carbonation responsible for the experimental behaviour at $\mathrm{CO}_{3}$ contents above $4 \%$, if there is any extra broadening at all in that region. For carbonate contents below $4 \%$ probably model IIIa (or IIIb) may be considered to be the most appropriate.

The perturbations in the lattice due to the incorporation of carbonate ions are shown to leave the spectral position of the phosphate line unchanged. This indicates that, at least with this basic simulation, the minimum of the potential function for the phosphate ion, when averaged over the whole environment, remains at the same $\mathrm{P}-0$ distance. This is in agreement with $\mathrm{x}$-ray results.

The results of the calculations offer some support to the suggestions in the literature that the dielectric constant has to be considered as a function of the distance in the lattice, increasing beyond the first environmental layer.

A comparison of the calculated and the experimental values for the line widths shows that there still are some discrepancies. Of course, the simulation is carried out

Table 4. Calculated phosphate line broadening $\left(\varepsilon_{r} \neq 1\right)$

\begin{tabular}{|c|c|c|c|c|}
\hline \multirow[b]{2}{*}{$\varepsilon_{r}$} & \multirow{2}{*}{$\begin{array}{l}\text { Substitution } \\
\text { model }\end{array}$} & \multicolumn{3}{|c|}{$\begin{array}{c}\Delta(\mathrm{FWHM}) / \% \mathrm{CO}_{3}\left(\mathrm{~cm}^{-1} / \%\right) \\
\text { a (see Table } 3)\end{array}$} \\
\hline & & $\alpha$ & $\beta$ & $\gamma$ \\
\hline $1 \rightarrow x$, at & 1 & $1.0^{\mathrm{a}}$ & 0.6 & 0.9 \\
\hline$r=0.55 \mathrm{~nm}$ & II & 4.1 & 4.0 & 4.2 \\
\hline$\alpha: x=1.25$ & IIIa & 1.7 & 2.2 & 2.2 \\
\hline$\beta: x=1.50$ & $\mathrm{IIIb}$ & 2.6 & 2.6 & 2.4 \\
\hline$y: x=2.00$ & IV & $0.9^{\mathrm{a}}$ & 2.2 & 1.4 \\
\hline $1+x r$ & 1 & 0.3 & 0.2 & 0.4 \\
\hline$r$ in $n m$; & II & 4.5 & 5.0 & 4.6 \\
\hline$\alpha: x=0.0$ & Illa & 1.7 & 2.0 & 2.4 \\
\hline$\beta: x=1.0$ & lllb & 2.7 & 2.3 & 2.0 \\
\hline$y: x=2.0$ & IV & 2.0 & $1.4^{\mathrm{a}}$ & 2.1 \\
\hline $\begin{array}{l}\text { ine shapes } \\
\text { ese line bro } \\
\text { ned in Tabl }\end{array}$ & $\begin{array}{l}\text { h cannot b } \\
\text { ing values }\end{array}$ & $\begin{array}{l}\text { cribed } \\
\text { bject }\end{array}$ & $\begin{array}{l}\text { igle l} \\
\text { ger er }\end{array}$ & heref \\
\hline
\end{tabular}


for a rigid lattice, and possible distortions of the lattice due to the substitutions and the creation of vacancies are not taken into account. Further, the periodic boundary conditions used in the calculations may be responsible for unwanted effects, such as a long-range ordering of the substitutions.

\section{CONCLUSIONS}

With a relatively simple inter- and intra-ionic potential function, consisting of a covalent and a Coulombic part, interactions can be calculated, leading to realistic results. The line width of the phosphate symmetrical stretching vibration is shown both theoretically and experimentally to be dependent on the carbonate content; the spectral position of the line does not shift on carbonation, either in the theory or the experiment. Several carbonate substitution models have been taken into account: the extra line broadening upon carbonation, calculated using the above-mentioned potential is in fairly good agreement with the experimental results. The calculated results are in favour of a substitution model proposed by Driessens et $a l^{18}$

\section{REFERENCES}

1. R. Z. LeGeros, J. P. LeGeros, O. R. Frantz and E. Klein, Dev. Appl. Spectrosc. 3 (1970).

2. J. Arends and C. L. Davidson, Calcif. Tissue Res 18, 65 (1975)

3. A. S. Hallsworth, J. A. Weatherell and C. Robinson, Caries Res. 7. 345 (1973).

4. F. F. M. De Mul, H. Buiteveld, J. Mud and J. Greve, J. Human Pathol. 15, 1062

5. F. F. M. De Mul, M. H. J. Hottenhuis, P. Bouter, J. Greve J. Arends and J. J. Ten Bosch, J. Dent. Res. 65, 437 (1986).

6. J. Arends, J. Schuthof, W. H. Van der Linden, P. Bennema and P. J. Van den Bert, J. Cryst. Growth 46, 213 (1979).

7. D. G. A. Nelson and B. E. Williamson, Aust. J. Chem. 35, 715 (1982).

8. W. B. White, in The Infrared Spectra of Minerals, edited by V. C. Farmer. Mineralogical Society, London (1974).

9. R. A. Young, Clin. Orthop. Relat. Res. 113, 249 (1975)

10. R. Wailaeys, in Proceedings of the Coloquium of the international Union of Pure and Applied Chemistry, Münster, pp. 185-190. Verlag Chemie, Weinheim (1954).

11. Y. Doi, T. Aoba, Y. Moriwaki, M. Okazaki and J. Takahashi, J. Dent. Res. 59, 1473 (1980).

12. D. McConnell, Am. Mineral. 23, 1 (1938).

13. D. McConneli, Naturwissenschaften 52, 183 (1952)

14. D. McConnell, Apatite. Springer-Verlag, Vienna (1973)

15. J. C. Labarthe, G. Bonel and G. Montel, Ann. Chim. (Paris) 8, 289 (1973)

16. J. C. Labarthe, M. Therase, G. Bonel and G. Montel, C. R. Acad Sci. Ser. C 276, 1175 (1973).
17. G. Montel, G. Bonel, J. G. Heughewaert, J. C. Trombe and C. Rey, J. Cryst. Growth 53, 74 (1981).

18. F. C. M. Driessens, R. M. H. Verbeeck and P. Kiekens, Z. Anorg Allg. Chem. 504, 195 (1983).

19. L. Pauling, The Nature of the Chemical Bond. Cornell University Press, Ithaca (1964).

20. G. McLennan and C. A. Beevers, Acta Crystallogr. 8, 579 (1955)

21. D. A. Long, Raman Spectroscopy. McGraw-Hill, New York (1977).

22. W. P. Griffith, in Infrared and Raman Spectroscopy of Luna and Terrestrial Minerals, edited by C. Karr, Jr., Academic Press, New York (1975).

23. G. Herzberg, Molecular Spectra and Molecular Structure. II Infrared and Raman Spectra of Polyatomic Molecules. Van Nostrand, Princeton, NJ (1945).

24. D. G. A. Nishino, S. Yamashita, T. Aoba, M. Okazaki and Y. Moriwaki, J. Dent. Res. 60, 751 (1981).

25. D. A. Greenberg, C. D. Barry and G. R. Marshall, J. Am. Chem. Soc. 100, 4020 (1978).

26. G. Wipff, P. Weiner and P. Kollman, J. Am. Chem. Soc. 104, 3250 (1982).

27. W. J. Colucci, R. D. Gandour and J. Mooberry, J. Am. Chem Soc. 108, 7142 (1986).

28. J. Uiterwijk, Complexation of Crown Ethers with Organic Molecules. Dissertation, University of Twente, Enschede, The Netherlands (1985) 